\title{
Minimum ranks of sign patterns via sign vectors and duality
}

\author{
Marina Arav, Frank J. Hall, Zhongshan Li, \\ Hein van der Holst, John Sinkovic, Lihua Zhang \\ Department of Mathematics and Statistics \\ Georgia State University \\ Atlanta, GA 30303, USA
}

\begin{abstract}
A sign pattern matrix is a matrix whose entries are from the set $\{+,-, 0\}$. The minimum rank of a sign pattern matrix $A$ is the minimum of the ranks of the real matrices whose entries have signs equal to the corresponding entries of $A$. It is shown in this paper that for any $m \times n$ sign pattern $A$ with minimum rank $n-2$, rational realization of the minimum rank is possible. This is done using a new approach involving sign vectors and duality. It is shown that for each integer $n \geq 9$, there exists a nonnegative integer $m$ such that there exists an $n \times m$ sign pattern matrix with minimum rank $n-3$ for which rational realization is not possible. A characterization of $m \times n$ sign patterns $A$ with minimum rank $n-1$ is given (which solves an open problem in Brualdi et al. [6]), along with a more general description of sign patterns with minimum rank $r$, in terms of sign vectors of certain subspaces. A number of results on the maximum and minimum numbers of sign vectors of $k$-dimensional subspaces of $\mathbb{R}^{n}$ are obtained. In particular, it is shown that the maximum number of sign vectors of 2 -dimensional subspaces of $\mathbb{R}^{n}$ is $4 n+1$. Several related open problems are stated along the way.
\end{abstract}

AMS classification: 15B35, 15B36, 52C40

Keywords: Sign pattern matrix, sign vectors, minimum rank, maximum rank, rational realization, duality

\footnotetext{
${ }^{*}$ Corresponding author, E-mail: marav@gsu.edu
} 


\section{Introduction}

An important part of the combinatorial matrix theory is the study of sign pattern matrices, which has been the focus of extensive research for the last 50 years ([7], [14]). A sign pattern matrix is a matrix whose entries are from the set $\{+,-, 0\}$. For a real matrix $B, \operatorname{sign}(B)$ is the sign pattern matrix obtained by replacing each positive (respectively, negative, zero) entry of $B$ by + (respectively,,- 0$)$. For a sign pattern matrix $A$, the sign pattern class (also known as the qualitative class) of $A$, denoted $Q(A)$, is defined as

$$
Q(A)=\{B: B \text { is a real matrix and } \operatorname{sign}(B)=A\} .
$$

The minimum rank of a sign pattern matrix $A$, denoted $\operatorname{mr}(A)$, is the minimum of the ranks of the real matrices in $Q(A)$. Determination of the minimum rank of a sign pattern matrix in general is a longstanding open problem (see [16]) in combinatorial matrix theory. Recently, there has been a number of papers concerning this topic, for example [1]-[11], [13]-[20]. In particular, matrices realizing the minimum rank of a sign pattern have applications in the study of neural networks [9] and communication complexity [20].

More specifically, in the study of communication complexity in computer science, $(+,-)$ sign pattern matrices arise naturally; the minimum rank (also known as the sign-rank) of the sign patterns plays an important role, as the minimum rank of a corresponding sign pattern matrix essentially determines the unbounded-error randomized communication complexity of a function. New results on the minimum ranks of sign pattern matrices would have significant impact on the area of communication complexity, as well as several areas of mathematics, such as matroid theory, polytope theory, computational geometry, graph theory and combinatorics [11].

In mathematics, rational realization is an important theme. For example, the study of the existence of rational (or integer) solutions of Diophantine equations is well-known. Steinitz's celebrated theorem stating that the combinatorial type of every 3-polytope is rationally realizable is a far-reaching result [22]. In combinatorics, rational realizability of certain point-line configurations is an important problem ([12, 21]); this is closely related to the realizability of the minimum rank of a sign pattern with minimum rank 3 [17]. More generally, the rational realizability of the minimum rank of a sign pattern matrix is equivalent to the rational realizability of a certain point-hyperplane configuration.

In [1], several classes of sign patterns $A$ for which rational realization of the minimum rank is guaranteed are identified, such as when $A$ is entrywise nonzero, or the minimum rank of $A$ is at most 2, or the minimum rank of $A$ is at least $n-1$, where $A$ is $m \times n$. It has been shown in [18], through the use of a result 
in projective geometry, that rational realization of the minimum rank is not always possible. Specifically, in [18], the authors showed that there exists a $12 \times 12$ sign pattern matrix with minimum rank 3 but there is no rational realization of rank 3 within the qualitative class of the sign pattern. Independently, Berman et al. [5] also provided an example of a sign pattern for which the rational minimum rank is strictly greater than the minimum rank over the reals. Both of these papers use techniques based on matroids. More recently, Jing et al. [17] found a $9 \times 9$ sign pattern matrix with minimum rank 3 whose rational minimum rank is 4 .

We note that Li et al. [19] showed that for every $n \times m$ sign pattern with minimum rank 2 , there is an integer matrix in its sign pattern class each of whose entries has absolute value at most $2 n-3$ that achieves the minimum rank 2 .

One goal of this paper is to show that for any $m \times n$ sign pattern $A$ with minimum rank $n-2$, rational realization of the minimum rank is possible. This is done using a new approach involving sign vectors and duality. Furthermore, it is shown that for each integer $n \geq 9$, there exists a nonnegative integer $m$ such that there exists an $n \times m$ sign pattern matrix with minimum rank $n-3$ for which rational realization is not possible.

Another goal is to use sign vectors of subspaces to investigate the minimum ranks of sign patterns further. In particular, a characterization of $m \times n$ sign patterns $A$ with minimum rank $n-1$ is given, which solves an open problem posed in Brualdi et al. [6]. We also obtain a characterization of L-matrices using sign vectors and a more general description of sign patterns with minimum rank $r$, in terms of sign vectors of certain subspaces. A number of results on the maximum and minimum numbers of sign vectors of $k$-dimensional subspaces of $\mathbb{R}^{n}$ are obtained. In particular, it is shown that the maximum number of sign vectors of 2-dimensional subspaces of $\mathbb{R}^{n}$ is $4 n+1$. Several related open problems are also discussed.

\section{Sign vectors and duality}

For any vector $x \in \mathbb{R}^{n}$, we define the sign vector of $x, \operatorname{sign}(x) \in\{+,-, 0\}^{n}$, by

$$
\operatorname{sign}(x)_{i}= \begin{cases}+ & \text { if } x_{i}>0 \\ 0 & \text { if } x_{i}=0 \\ - & \text { if } x_{i}<0\end{cases}
$$

For any subspace $L \subseteq \mathbb{R}^{n}$, we define the set of sign vectors of $L$ as

$$
\operatorname{sign}(L)=\{\operatorname{sign}(x) \mid x \in L\} .
$$

Observation 1. If $K$ and $L$ are subspaces of $\mathbb{R}^{n}$ with $\operatorname{sign}(K)=\operatorname{sign}(L)$, then $\operatorname{dim}(K)=\operatorname{dim}(L)$. 
Indeed, consider the sign vectors of the columns of the reduced column echelon form of a matrix whose columns form a basis of $K$. Such sign vectors are in $\operatorname{sign}(K)$ and hence also in $\operatorname{sign}(L)$. It follows that $\operatorname{dim}(L) \geq \operatorname{dim}(K)$. By reversing the roles of $K$ and $L$, we get the reverse inequality.

For a subspace $L \subseteq \mathbb{R}^{n}$, as usual, $L^{\perp}=\left\{x \in \mathbb{R}^{n} \mid x^{T} y=0\right.$ for all $\left.y \in L\right\}$ denotes the orthogonal complement of $L$.

Two sign vectors $c, x \in\{+,-, 0\}^{n}$ are said to be orthogonal, written as $c \perp x$, if one of the following two conditions holds:

1. for each $i$, we have $c_{i}=0$ or $x_{i}=0$, or

2. there are indices $i, j$ with $c_{i}=x_{i} \neq 0$ and $c_{j}=-x_{j} \neq 0$.

For a set of sign vectors $S \subseteq\{+,-, 0\}^{n}$, the orthogonal complement of $S$ is

$$
S^{\perp}=\left\{c \in\{+,-, 0\}^{n} \mid c \perp x=0 \text { for all } x \in S\right\} .
$$

Notice that if $c, x \in \mathbb{R}^{n}$ and $c^{T} x=0$, then $\operatorname{sign}(c) \perp \operatorname{sign}(x)=0$.

We will use the following theorem, a proof of which can be found in Ziegler [22].

Theorem 2 (Duality of oriented matroids). For any subspace $L \subseteq \mathbb{R}^{n}$,

$$
\operatorname{sign}(L)^{\perp}=\operatorname{sign}\left(L^{\perp}\right) .
$$

A subspace $L \subseteq \mathbb{R}^{n}$ is called rational if $L$ has a basis consisting of rational vectors.

Lemma 3. Let $L$ be a rational subspace of $\mathbb{R}^{n}$ and let $B=\left[\begin{array}{llll}b_{1} & b_{2} & \ldots & b_{k}\end{array}\right]$ be a matrix whose columns form a rational basis for $L$. Then for any $s \in \operatorname{sign}(L)$, there exists a rational vector $x \in \mathbb{Q}^{k}$ such that $\operatorname{sign}(B x)=s$.

Proof. Let $N=\left\{i: s_{i}=0\right\}$ and let $V=\left\{z \in \mathbb{R}^{k}:(B z)_{i}=0\right.$ for all $\left.i \in N\right\}$. There exists a vector $y \in V$ such that $\operatorname{sign}(B y)=s$. Since $\left\{b_{1}, b_{2}, \ldots, b_{k}\right\}$ is a rational basis for $L, V$ has a rational basis $\left\{c_{1}, c_{2}, \ldots, c_{t}\right\}$. Let $a=\left[\begin{array}{llll}a_{1} & a_{2} & \ldots & a_{t}\end{array}\right]^{T} \in$ $\mathbb{R}^{t}$ such that $a_{1} c_{1}+a_{2} c_{2}+\cdots+a_{t} c_{t}=y$. There exists a sequence of rational vectors $a(n)=\left[\begin{array}{llll}a_{1}(n) & a_{2}(n) & \ldots & a_{t}(n)\end{array}\right]^{T}, n \in \mathbb{N}$ such that $\lim _{n \rightarrow \infty} a(n)=a$. Define $y(n)=a_{1}(n) c_{1}+a_{2}(n) c_{2}+\cdots+a_{t}(n) c_{t}$. Then $\lim _{n \rightarrow \infty} y(n)=y$ and each $y(n)$ is a rational vector. Since $\lim _{n \rightarrow \infty} B y(n)=B y$, there exists an $m$ such that $\operatorname{sign}(B y(m))=\operatorname{sign}(B y)=s$. By letting $x=y(m)$, the statement of the lemma is proved. 
Theorem 4. Let $n$ and $r$ be nonnegative integers. Rational realization of the minimum rank is always possible for all $n \times m$ sign pattern matrices with minimum rank $r$ if and only if for each subspace $L \subseteq \mathbb{R}^{n}$ with dimension $r$ there exists a rational subspace $K \subseteq \mathbb{R}^{n}$ with $\operatorname{dim}(K)=r$ and $\operatorname{sign}(K)=\operatorname{sign}(L)$.

Proof. Suppose that rational realization of the minimum rank is always possible for all $n \times m$ sign pattern matrices with minimum rank $r$. Let $L \subseteq \mathbb{R}^{n}$ with dimension $r$. Let $s_{1}, \ldots, s_{k}$ be all sign vectors in $\operatorname{sign}(L)$. Let $A$ be the $n \times k$ sign pattern matrix whose $i$ th column is $s_{i}$. Then $\operatorname{mr}(A) \leq r$, as for each sign vector $s_{i}$ we can choose a vector $x_{i} \in L$ with $\operatorname{sign}\left(x_{i}\right)=s_{i}$, and the matrix $B$, whose $i$ th column is $x_{i}$, has rank $r$. Thus, there exists a rational matrix $C \in Q(A)$ with rank at most $r$. Let $K$ be the column space of $C$. Then $\operatorname{sign}(K)=\operatorname{sign}(L)$ and $K$ is rational. Since $\operatorname{sign}(K)=\operatorname{sign}(L)$, by Observation $1, \operatorname{dim}(K)=r$.

Conversely, suppose that for each subspace $L \subseteq \mathbb{R}^{n}$ with dimension $r$ there exists a rational subspace $K \subseteq \mathbb{R}^{n}$ with $\operatorname{sign}(K)=\operatorname{sign}(L)$. Let $A$ be an $n \times m$ sign pattern matrix with $\operatorname{mr}(A)=r$ and let $F \in Q(A)$ with $\operatorname{rank}(F)=r$. There exist an $n \times r$ matrix $U$ and an $r \times m$ matrix $V$ such that $F=U V$. Let $v_{i}$ denote the $i$ th column of $V$. Notice that $\operatorname{sign}\left(U v_{i}\right)$ is the $i$ th column of $A$. Let $L$ be the subspace of $\mathbb{R}^{n}$ spanned by the columns of $U$. By assumption, there exists a rational subspace $K$ of $\mathbb{R}^{n}$ such that $\operatorname{sign}(L)=\operatorname{sign}(K)$. Let $\left\{b_{1}, b_{2}, \ldots, b_{r}\right\}$ be a rational basis for $K$ and let $B=\left[\begin{array}{llll}b_{1} & b_{2} & \ldots & b_{r}\end{array}\right]$. Since $\operatorname{sign}(L)=\operatorname{sign}(K)$, there exist rational vectors $x_{i} \in \mathbb{Q}^{r}, i=1,2, \ldots, m \operatorname{such}$ that $\operatorname{sign}\left(B x_{i}\right)=\operatorname{sign}\left(U v_{i}\right)$, by Lemma 3 . Let $C=B\left[\begin{array}{llll}x_{1} & x_{2} & \ldots & x_{m}\end{array}\right]$. Then $C \in Q(A), \operatorname{rank} C \leq r$, and $C$ is a rational matrix. Since $\operatorname{mr}(A)=r, \operatorname{rank} C=r$.

The next theorem can be found in [1] and [19].

Theorem 5. Rational realization of the minimum rank is always possible for every $n \times m$ sign pattern matrix $A$ with minimum rank 2 .

The following theorem follows from the two preceding theorems.

Theorem 6. For any subspace $L \subseteq \mathbb{R}^{n}$ with $\operatorname{dim}(L)=2$, there exists a rational subspace $K \subseteq \mathbb{R}^{n}$ with $\operatorname{dim}(K)=2$ and $\operatorname{sign}(K)=\operatorname{sign}(L)$.

Since the solution space of a system of homogeneous linear equations with rational coefficients has a rational basis, it is clear that if $L \subseteq \mathbb{R}^{n}$ is a rational subspace, then $L^{\perp}$ is also a rational subspace.

Lemma 7. For any subspace $L \subseteq \mathbb{R}^{n}$ with $\operatorname{dim} L=n-2$, there exists a rational subspace $K \subseteq \mathbb{R}^{n}$ with $\operatorname{dim} K=n-2$ such that $\operatorname{sign}(K)=\operatorname{sign}(L)$. 
Proof. By Theorem 6, there exists a rational subspace $M \subseteq \mathbb{R}^{n}$ with $\operatorname{dim} M=2$ such that $\operatorname{sign}\left(L^{\perp}\right)=\operatorname{sign}(M)$. Let $K=M^{\perp}$. Then $K$ is a rational subspace of $\mathbb{R}^{n}$ with $\operatorname{dim} K=n-2$ and, by Theorem $2, \operatorname{sign}(K)=\operatorname{sign}\left(M^{\perp}\right)=$ $\operatorname{sign}(M)^{\perp}=\operatorname{sign}\left(L^{\perp}\right)^{\perp}=\operatorname{sign}(L)$. result.

By Theorem 4, and by considering the transpose if needed, we get the following

Theorem 8. Rational realization of the minimum rank is always possible for $n \times m$ sign pattern matrices with minimum rank $n-2$ or $m-2$.

From the $9 \times 9$ example given in [17], the next theorem follows immediately.

Theorem 9. For each integer $n \geq 9$, there exists an $n \times m$ sign pattern matrix $A$ with $\operatorname{mr}(A)=3$ for which no rational realization is possible.

From Theorem 4 we then obtain the following corollary.

Corollary 10. Let $n \geq 9$ be an integer. Then there exists a subspace $L \subseteq \mathbb{R}^{n}$ with $\operatorname{dim} L=3$ such that there is no rational subspace $K \subseteq \mathbb{R}^{n}$ with $\operatorname{dim} K=3$ and $\operatorname{sign}(L)=\operatorname{sign}(K)$.

The following result then follows from Theorem 2

Lemma 11. Let $n \geq 9$ be an integer. There exists a subspace $M \subseteq \mathbb{R}^{n}$ with $\operatorname{dim} M=n-3$ such that there is no rational subspace $K \subseteq \mathbb{R}^{n}$ with $\operatorname{dim} K=$ $n-3$ and $\operatorname{sign}(K)=\operatorname{sign}(M)$.

Proof. By Corollary 10 , there exists a subspace $L \subseteq \mathbb{R}^{n}$ with $\operatorname{dim} L=3$ such that there is no rational subspace $K \subseteq \mathbb{R}^{n}$ with $\operatorname{dim} K=3$ and $\operatorname{sign}(L)=\operatorname{sign}(K)$. Let $M=L^{\perp}$. Then there is no rational subspace $K \subseteq \mathbb{R}^{n}$ with $\operatorname{dim} K=n-3$ and $\operatorname{sign}(K)=\operatorname{sign}(M)$.

Another application of Theorem 4 gives the following fact.

Theorem 12. For each integer $n \geq 9$, there exists a nonnegative integer $m$ such that there exists an $n \times m$ sign pattern matrix with minimum rank $n-3$ for which rational realization is not possible.

However, this leaves open the following question.

Problem 13. Is it true that for each integer $n \geq 9$, there exists an $n \times n$ sign pattern matrix with minimum rank $n-3$ for which rational realization is not possible?

Another natural question is the following. 
Problem 14. Is it true that for every 3 dimensional subspace $L$ of $\mathbb{R}^{8}$, there is a rational subspace $K$ such that $\operatorname{sign}(L)=\operatorname{sign}(K)$ ?

There are connections between rational realization of the minimum ranks of sign patterns and the existence of rational solutions of certain matrix equations, as indicated in [3]. Using this connection and Theorem 8 for the case of $\operatorname{mr}(A)=$ $n-2$, we are able to prove the following result.

Theorem 15. Suppose that $B, C$ and $E$ are real matrices such that $B C=E$. If either $E$ has 2 rows or 2 columns, then there exist rational matrices $\tilde{B}, \tilde{C}$ and $\tilde{E}$ $\operatorname{such}$ that $\operatorname{sign}(\tilde{B})=\operatorname{sign}(B), \operatorname{sign}(\tilde{C})=\operatorname{sign}(C), \operatorname{sign}(\tilde{E})=\operatorname{sign}(E)$, and $\tilde{B} \tilde{C}=$ $\tilde{E}$.

Proof. Without loss of generality, assume that $E$ has two columns. Consider the $2 \times 2$ block matrix

$$
M=\left[\begin{array}{cc}
I_{n} & C \\
B & E
\end{array}\right] .
$$

Observe that $M$ has $n+2$ columns and the Schur complement of $I_{n}$ in $M$ is 0 . Hence, $\operatorname{rank}(M)=n$. It follows that the minimum rank of the sign pattern $\operatorname{sign}(M)$ is $n$. Hence, from Theorem 8 there is a rational matrix

$$
\tilde{M}=\left[\begin{array}{cc}
D & C_{1} \\
B_{1} & E_{1}
\end{array}\right]
$$

of rank $n$ in $Q(\operatorname{sign}(M))$.

It follows that the Schur complement of $D$ in $\tilde{M}$ is $E_{1}-B_{1} D^{-1} C_{1}=0$. The rational matrices $\tilde{B}=B_{1}, \tilde{C}=D^{-1} C_{1}$ and $\tilde{E}=E_{1}$ clearly satisfy the desired properties.

Since the zero entries of the matrix $E$ in a real matrix equation $B C=E$ are the major obstructions for the existence rational solutions within the same corresponding sign pattern classes, the preceding theorem suggests the following conjecture.

Conjecture 16. Let $B, C$ and $E$ be real matrices such that $B C=E$. If all the zero entries of $E$ are contained in a submatrix with either 2 rows or 2 columns, then there exist rational matrices $\tilde{B}, \tilde{C}$, and $\tilde{E}$, such that $\operatorname{sign}(\tilde{B})=\operatorname{sign}(B), \operatorname{sign}(\tilde{C})=$ $\operatorname{sign}(C), \operatorname{sign}(\tilde{E})=\operatorname{sign}(E)$, and $\tilde{B} \tilde{C}=\tilde{E}$.

\section{Further results on minimum ranks and sign vectors of subspaces}

We now investigate the minimum ranks of sign patterns further using sign vectors and duality. 
Similar to the concept of $\operatorname{mr}(A)$, the maximum rank of a sign pattern matrix $A$, denoted $\operatorname{MR}(A)$, is the maximum of the ranks of the real matrices in $Q(A)$. It is well-known that $\operatorname{MR}(A)$ is the maximum number of nonzero entries of $A$ with no two of the nonzero entries in the same row or in the same column. By a theorem of Konig, the minimal number of lines (namely, rows and columns) in $A$ that cover all of the nonzero entries of $A$ is equal to the maximal number of nonzero entries in $A$, no two of which are on the same line. This common number is also called the term rank [14]. In contrast to the rational realization problem of the minimum rank, we note that through diagonal dominance, it can be easily seen that for every sign pattern matrix $A, \operatorname{MR}(A)$ can always be achieved by a rational matrix.

Sign pattern matrices $A$ that require a unique rank (namely, $\operatorname{MR}(A)=\operatorname{mr}(A)$ ) were characterized by Hershkowitz and Schneider in [15].

It is shown in [2] that rational realization of the minimum rank of $A$ is always possible if $\operatorname{MR}(A)-\operatorname{mr}(A)=1$. The following conjecture from [2] remains open.

Conjecture 17. [2] For every sign pattern matrix $A$ with $M R(A)-\operatorname{mr}(A)=2$, there is a rational realization of the minimum rank.

In [2], the study of sign patterns $A$ with $\operatorname{MR}(A)-\operatorname{mr}(A)=1$ is reduced to the study of $m \times n$ sign patterns $A$ such that $\operatorname{MR}(A)=n$ and $\operatorname{mr}(A)=n-1$. In this connection, using sign vectors and duality, we obtain the following characterization of the $m \times n$ sign patterns $A$ such that $\operatorname{MR}(A)=n$ and $\operatorname{mr}(A)=n-1$ (which was raised as an open problem in [6]). Since the condition $\operatorname{MR}(A)=n$ is easily checked, it suffices to characterize $m \times n$ sign patterns $A$ with $\operatorname{mr}(A)=n-1$.

Theorem 18. Let $A$ be an $m \times n$ sign pattern and let row $(A)$ denote the set of the row vectors of $A$. Then $\operatorname{mr}(A)=n-1$ if and only if the following two conditions hold.

(a). There is a nonzero sign vector $x \in\{+,-, 0\}^{n}$ such that $\operatorname{row}(A) \subseteq x^{\perp}$.

(b). For every two dimensional subspace $L$ of $\mathbb{R}^{n}, \operatorname{row}(A) \nsubseteq \operatorname{sign}(L)^{\perp}$.

Proof. Assume that $\operatorname{mr}(A)=n-1$. Then there is a real matrix $A_{1} \in Q(A)$ such that $\operatorname{rank}\left(A_{1}\right)=n-1$. Let $v_{0}$ be a nonzero vector in the null space $\operatorname{Null}\left(A_{1}\right)$ and let $x=\operatorname{sign}\left(v_{0}\right)$. Then it is clear that $\operatorname{row}(A) \subseteq x^{\perp}$, namely, (a) holds. Further, consider any two dimensional subspace $L$ of $\mathbb{R}^{n}$. Suppose that $\operatorname{row}(A) \subseteq$ $\operatorname{sign}(L)^{\perp}$. Then by Theorem 2, $\operatorname{row}(A) \subseteq \operatorname{sign}\left(L^{\perp}\right)$. Since $L^{\perp}$ is a subspace of dimension $n-2$ and every row vector of $A$ is contained in $\operatorname{sign}\left(L^{\perp}\right)$, it is clear that the span of the real vectors in $L^{\perp}$ whose sign vectors agree with the rows of $A$ is a subspace of dimension at most $n-2$, that is to say, there is a real matrix $A_{2} \in Q(A)$ of rank at most $n-2$, which contradicts the assumption that $\operatorname{mr}(A)=n-1$. 
Conversely, assume that (a) and (b) hold. Let $x_{1}$ be the $(1,-1,0)$ vector in $Q(x)$. From (a), it is easily seen that there is a real matrix $A_{1} \in Q(A)$ such that $A_{1} x_{1}=0$. It follows that $\operatorname{rank}\left(A_{1}\right) \leq n-1$ and hence $\operatorname{mr}(A) \leq n-1$. Suppose that $\operatorname{mr}(A) \leq n-2$. Then there is a matrix $A_{2} \in Q(A)$ with $\operatorname{rank}\left(A_{2}\right) \leq n-2$. It follows that $\operatorname{Null}\left(A_{2}\right)$ has dimension at least 2 . Hence, $\operatorname{Null}\left(A_{2}\right)$ contains a subspace $L \in \mathbb{R}^{n}$ of dimension 2. It follows that $\operatorname{row}(A) \subseteq \operatorname{sign}\left(\operatorname{Null}\left(A_{2}\right)\right)^{\perp} \subseteq$ $\operatorname{sign}(L)^{\perp}$, contradiction (b). Thus $\operatorname{mr}(A)=n-1$.

We note that for an $m \times n$ sign pattern matrix $A$, the condition that each row vector of $A$ is in $\{u, v\}^{\perp}$ for some two nonzero sign vectors $u, v \in\{+,-, 0\}^{n}$ with $u \neq \pm v$ does not imply that $\operatorname{mr}(A) \leq n-2$, as the following example shows.

Example 19. Let $A=\left[\begin{array}{lll}+ & + & + \\ 0 & + & +\end{array}\right], u=\left[\begin{array}{c}+ \\ + \\ -\end{array}\right]$, and $v=\left[\begin{array}{c}0 \\ + \\ -\end{array}\right]$. It is obvious that each row vector of $A$ is in $\{u, v\}^{\perp}$ for the two nonzero sign vectors $u, v \in$ $\{+,-, 0\}^{3}$ with $u \neq \pm v$. But clearly, $\operatorname{mr}(A)=2$, so $\operatorname{mr}(A) \not \leq n-2=1$.

Using a similar argument as in the proof of Theorem 18, we can show the following more general result that characterizes sign patterns with minimum rank $r$, for each possible $r \geq 1$.

Theorem 20. Let $A$ be an $m \times n$ sign pattern and let row $(A)$ denote the set of the row vectors of $A$, and let $r$ be a any integer such that $1 \leq r \leq \min \{m, n\}$. Then $m r(A)=r$ if and only if the following two conditions hold.

(a). There is a subspace $L \subseteq \mathbb{R}^{n}$ with $\operatorname{dim}(L)=r$ such that $\operatorname{row}(A) \subseteq \operatorname{sign}(L)$.

(b). For every subspace $V$ of $\mathbb{R}^{n}$ with $\operatorname{dim}(L)=r-1$, row $(A) \nsubseteq \operatorname{sign}(V)$.

In particular, the above theorem gives a characterization of L-matrices (namely, $m \times n$ sign patterns $A$ with $\operatorname{mr}(A)=n)$. Note that every subset of $\{+,-, 0\}^{n}$ is of course contained in $\operatorname{sign}\left(\mathbb{R}^{n}\right)$ and for every subspace $V$ of $\mathbb{R}^{n}$ of dimension $n-1$, $\operatorname{sign}(V)=\operatorname{sign}(x)^{\perp}$ for a nonzero vector $x$ in $V^{\perp}$.

Corollary 21. Let $A$ be an $m \times n$ sign pattern and let row $(A)$ denote the set of the rows of $A$. Then $m r(A)=n$ if and only if for every nonzero sign vector $x \in\{+,-, 0\}^{n}, \operatorname{row}(A) \nsubseteq x^{\perp}$.

The last characterization of L-matrices can be seen to be equivalent to the characterization of such matrices found in [7] (where a sign pattern is defined to be an L-matrix if the minimum rank is equal to its number of rows). 
Let $S_{k, n}$ (respectively, $s_{k, n}$ ) denote the maximum cardinality (respectively, minimum cardinality) of $\operatorname{sign}(L)$ as $L$ runs over all $k$ dimensional subspaces of $\mathbb{R}^{n}$. In other words,

$$
S_{k, n}=\max _{\substack{L \subseteq \mathbb{R}^{n} \\ \operatorname{dim}(L)=k}}|\operatorname{sign}(L)|, \quad s_{k, n}=\min _{\substack{L \subseteq \mathbb{R}^{n} \\ \operatorname{dim}(L)=k}}|\operatorname{sign}(L)| .
$$

For every subspace $L$, as the nonzero vectors in $\operatorname{sign}(L)$ occur in disjoint pairs of vectors that are negatives of each other, it is clear that $|\operatorname{sign}(L)|$ is odd. Thus $S_{k, n}$ and $s_{k, n}$ are always odd. For each $k(0 \leq k \leq n-1)$, since every $k$ dimensional subspace of $\mathbb{R}^{n}$ is contained in a subspace of dimension $k+1$, it is clear that $S_{k, n} \leq S_{k+1, n}$ and $s_{k, n} \leq s_{k+1, n}$.

Obviously, $S_{0, n}=s_{0, n}=1, S_{1, n}=s_{1, n}=3$, and $S_{n, n}=s_{n, n}=3^{n}$. For a nonzero sign vector $x \in\{+,-, 0\}^{n}$ with exactly $t$ nonzero entries, it can be seen that $\left|x^{\perp}\right|=3^{n-t}\left(3^{t}-2\left(2^{t}-1\right)\right)$, which attains its maximum $3^{n}-2\left(2^{n}-1\right)$ when $t=n$ and attains its minimum $3^{n-1}$ when $t=1$. Thus by the duality theorem, we get $S_{n-1, n}=3^{n}-2\left(2^{n}-1\right)$ and $s_{n-1, n}=3^{n-1}$. In particular, for $n=3$, the above argument shows that the only possible cardinalities of $|\operatorname{sign}(L)|$ for 2-dimensional subspaces of $\mathbb{R}^{3}$ are 9 and 13 .

By considering the reduced row echelon form of a matrix whose rows form a basis for a $k$-dimensional subspace $L$ of $\mathbb{R}^{n}$ and observing that the components in the pivot columns of the sign vectors of the vectors $L$ are independent and arbitrary, it can be seen that $s_{k, n} \geq 3^{k}$, for each $k, 1 \leq k \leq n$. For the $k$-dimensional subspace $L$ spanned by the standard vectors $e_{1}, e_{2}, \ldots, e_{k}$, it can be seen that equality in the last inequality can be achieved. Thus, $s_{k, n}=3^{k}$, for each $k, 1 \leq k \leq n$. We record some of these results below.

Theorem 22. Let $n \geq 2$. Then $S_{n-1, n}=3^{n}-2\left(2^{n}-1\right)$, and $s_{k, n}=3^{k}$, for each $k, 1 \leq k \leq n$.

To determine $S_{2, n}$, we need some terminology and two results on sign patterns with minimum rank 2 given in [19]. A sign pattern is said to be condensed if does not contain a zero row or zero column and no two rows or two columns are identical or are negatives of each other. Clearly, given any nonzero sign pattern $A$, we can delete zero, duplicate or opposite rows and columns of $A$ to get a condensed sign pattern matrix $A_{c}$ (called the condensed sign pattern of $A$ ). Obviously, for any sign pattern $A, \operatorname{mr}(A)=\operatorname{mr}\left(A_{c}\right)$.

Theorem 23. [19] For any sign pattern $A, m r(A)=2$ if and only if $A_{c}$ has at least two rows, each row of $A_{c}$ has at most one zero entry, and there exist a permutation sign pattern $P$ and a signature sign pattern $D$ such that each row of $A_{c} D P$ is monotone (in the sense that $-\leq 0 \leq+$ ). 
Corollary 24. Let $\mathcal{A}$ be an $m \times n$ condensed sign pattern with minimum rank 2 . Then $m \leq 2 n$.

We now determine the extremal cardinalities of $\operatorname{sign}(V)$ for two dimensional subspaces $V$ of $\mathbb{R}^{n}(n \geq 2)$.

Theorem 25. For each $n \geq 2, S_{2, n}=4 n+1$.

Proof. Let $V$ be any subspace of $\mathbb{R}^{n}$ with $\operatorname{dim}(V)=2$. Let $A$ be the sign pattern whose rows are the sign vectors in $\operatorname{sign}(V)$. Then clearly $\operatorname{mr}(A)=2$. Note that $A$ has a zero row and the nonzero rows of $A$ occur in pairs of vectors that are negatives of each other. By deleting the zero row and choosing a representative out of each nonzero pair and possibly deleting some columns of $A$, we get the condensed sign pattern $A_{c}$ of $A$. By the preceding corollary, the number of rows of $A_{c}$ is at most $2 n_{1}$, where $n_{1}$ is the number of columns of $A_{c}$. It is then clear that $|\operatorname{sign}(V)| \leq 4 n_{1}+1 \leq 4 n+1$.

By considering the $2 n \times n$ sign pattern $B=\left[\begin{array}{l}T_{1} \\ T_{2}\end{array}\right]$, where

$$
T_{1}=\left[\begin{array}{cccc}
0 & + & \cdots & + \\
- & 0 & \ddots & \vdots \\
\vdots & \ddots & \ddots & + \\
- & \cdots & - & 0
\end{array}\right], \quad \text { and } \quad T_{2}=\left[\begin{array}{cccc}
- & + & \cdots & + \\
- & - & \ddots & \vdots \\
\vdots & \ddots & \ddots & + \\
- & \cdots & - & -
\end{array}\right] \text {, }
$$

it can be seen from Theorem 23 that $\operatorname{mr}(B)=2$ and the equality $|\operatorname{sign}(V)|=$ $4 n+1$ can be achieved.

Due to the lack of a simple structural characterization of sign patterns with minimum rank 3 comparable to Theorem 23, determining $S_{3, n}$ seems to be a difficult problem. We note that by considering the direct sum $[+] \oplus B$, where $B$ is the $2(n-1) \times(n-1)$ sign pattern with $\operatorname{mr}(B)=2$ as in the preceding proof, it can be seen that

$$
S_{3, n} \geq 3(4(n-1)+1)=3(4 n-3) .
$$

Problem 26. Find sharp bounds on $S_{k, n}$, for $3 \leq k \leq n-2$.

\section{References}

[1] M. Arav, F.J. Hall, S. Koyuncu, Z. Li, B. Rao, Rational realizations of the minimum rank of a sign pattern matrix, Linear Algebra Appl. 409 (2005), $111-125$. 
[2] M. Arav, F. J. Hall, Z. Li, A. Merid and Y. Gao, Sign Patterns that Require Almost Unique Rank, Linear Algebra Appl. 430 (2009), 7-16.

[3] M. Arav, F. Hall, Z. Li, and B. Rao, Rational solutions of certain matrix equations, Linear Algebra Appl. 430 (2009), 660-663.

[4] F. Barioli, S. M. Fallat, H. T. Hall, D. Hershkowitz, L. Hogben, H. van der Holst, B. Shader, On the minimum rank of not necessarily symmetric matrices: a preliminary study, Electron. J. Linear Algebra 18 (2009), 126-145.

[5] A. Berman, S. Friedland, L. Hogben, U.G. Rothblum, B. Shader, Minimum rank of matrices described by a graph or pattern over the rational, real and complex numbers, The Electron. J. Combin. 15 (2008), 19 pp.

[6] R. Brualdi, S. Fallat, L. Hogben, B. Shader, and P. van den Driessche, Final report: Workshop on Theory and Applications of Matrices described by Patterns, Banff International Research Station, Jan. 31-Feb. 5, 2010.

[7] R.A. Brualdi, B.L. Shader, Matrices of Sign-Solvable Linear Systems, Cambridge University Press, Cambridge, 1995.

[8] G. Chen, F.J. Hall, Z. Li, B. Wei, On ranks of matrices associated with trees, Graphs Combin. 19 (2003), 323-334.

[9] P. Delsarte, Y. Kamp, Low rank matrices with a given sign pattern, SIAM J. Discrete Math. 2 (1989), 51-63.

[10] J. Forster, A linear lower bound on the unbounded error probabilistic communication complexity, J. Comput. Syst. Sci. 65(4) (2002), 612-625.

[11] R.L. Graham, M. Grötschel and L. Lovász (Editors), Handbook of Combinatorics, MIT Press, 1996

[12] B. Grünbaum, Configurations of Points and Lines, AMS, 2009.

[13] F.J. Hall, Z. Li, B. Rao, From Boolean to sign pattern matrices, Linear Algebra Appl. 393 (2004), 232-251.

[14] F.J. Hall, Z. Li, Sign Pattern Matrices, in Handbook of Linear Algebra (L. Hogben et al., editors), Chapman and Hall/CRC Press, Boca Raton, 2013.

[15] D. Hershkowitz, H. Schneider, Ranks of zero patterns and sign patterns, Linear and Multilinear Algebra 34 (1993), 3-19. 
[16] C.R. Johnson, Some outstanding problems in the theory of matrices, Linear and Multilinear Algebra 12 (1982), 99-108.

[17] G. Jing, W. Gao, Y. Gao, F. Gong, Z. Li, Y. Shao, Lihua Zhang, Sign patterns with minimum rank 3 and point-line configurations on the plane, preprint [arXiv??]

[18] S. Kopparty, K.P.S. Bhaskara Rao, The minimum rank problem: a counterexample, Linear Algebra Appl. 428 (2008), 1761-1765.

[19] Z. Li, Y. Gao, M. Arav, F. Gong, W. Gao, F. J.Hall, H. van der Holst, Sign patterns with minimum rank 2 and upper bounds on minimum ranks, Linear and Multilinear Algebra 61 (2013), 895-908.

[20] A.A. Razborov, A.A. Sherstov, The sign rank of $\mathrm{AC}^{0}$, SIAM Journal of Computing 39 (2010), 1833-1855.

[21] B. Sturmfels and N. White, All $11_{3}$ and $12_{3}$-configurations are rational, Aequationes Mathematicae 39 (1990), 254-260

[22] G.M. Ziegler, Lectures on Polytopes, Springer-Verlag, NY, 1994. 